\title{
Measuring Velocity of Moving Inertial Frames with Light Transmissions
}

\author{
Steven D. Deines \\ Donatech Corporation, Inc., Fairfield, USA
}

\section{Email address:}

sddeines@hotmail.com

\section{To cite this article:}

Steven D. Deines. Measuring Velocity of Moving Inertial Frames with Light Transmissions. International Journal of Applied Mathematics and Theoretical Physics. Vol. 3, No. 3, 2017, pp. 56-60. doi: 10.11648/j.ijamtp.20170303.13

Received: August 31,2016; Accepted: November 26, 2016; Published: May 6, 2017

\begin{abstract}
Newton's Mathematical Principles of Natural Philosophy provided the foundation of classical physics. This paper reviews several of his critical definitions, his three axioms, key corollaries, and concept of inertial frames. Newton's first axiom or law requires the vector addition of velocities by Corollary I to translate the equation of motion of a constantly moving body from one inertial frame to another inertial frame. His relativity principle in Corollary $\mathrm{V}$ is often expanded to mean that any equation retains the same form in all inertial frames. This is true if the equations involve only Newtonian forces, but equations that specify velocity need to include the mutual velocity between moving inertial frames to fully transform the results between all reference frames. The speed of light parameter must correctly incorporate the mutual velocity between moving inertial frames. It is assumed the speed of light is a constant in all directions only in absolutely stationary, nongravitated reference frames, which is less restrictive than the assumption of universal speed of light in all inertial frames. A test is outlined with suggested equipment to measure the one-way speed of light simultaneously in three dimensions. Equations are provided to convert the results into the instantaneous directional velocity of the laboratory frame. It may take a few years to collect data to separate the Earth's rotation, precession, nutation, polar wobble, Earth's orbital velocity around the Earth-Sun and Earth-Moon barycenters, and the solar system's movement due to the Milky Way's rotation and translational velocity within the universe.
\end{abstract}

Keywords: Simultaneity, Relativity, Timing, Speed of Light

\section{Introduction}

Isaac Newton established the foundations of mechanical physics in his Mathematical Principles of Natural Philosophy (i.e. Principia from the Latin edition) in 1687 [1]. He made it clear that only relative motions were measurable in his Definition section: "And so, instead of absolute places and motions, we use relative ones; and that without any inconvenience in common affairs; we ought to...consider things themselves, distinct from what are only sensible measures of them. For it may be that there is nobody really at rest, to which the places and motions of others may be referred.... Upon which account, the true and absolute motion of a body cannot be determined by the translation of it from those which only seem to rest; for the external bodies ought not only to appear as rest, but to be really at rest.... Now no other places are immovable but those that, from infinity to infinity, do all retain the same given position one to another; and upon this account must ever remain unmoved; and do thereby constitute immovable space." [1] Newton defined motion in Definition II [1] as "The quantity of motion [now called momentum] is the measure of the same, arising from the velocity and quantity of matter conjointly" (i.e. momentum $=$ mass $\mathrm{x}$ velocity). Then, Newton stated his three laws in the Axiom section [1]:

Law I. Every body continues in its state of rest, or of uniform motion in a right line, unless it is compelled to change that state by forces impressed upon it.

Law II. The change of motion (i.e. derivative of momentum) is proportional to the motive force impressed; and is made in the direction of the right line in which that force is impressed.

Law III. To every action there is always opposed an equal reaction: or, the mutual actions of two bodies upon each other are always equal, and directed to contrary parts.

He described two forces simultaneously acting on a body would be equivalent to the diagonal vector of a parallelogram 
with the sides being the separate vector forces (Corollary I), which defines vector addition of forces. In the environment that we can measure, Newton considered bodies as movable, so all relationships between observed bodies are relative, except the abstraction that places infinitely away may be considered absolutely stationary. Several times in his Definitions section, he described that external forces applied to internal bodies of a system would have no effect on the relative motion (i.e. momentum) of those internal bodies. $\mathrm{He}$ specified in the Axioms or Laws of Motion section this concept in Corollary VI of the Axioms section as "If bodies, moved in any manner among themselves, are urged in the direction of parallel lines by equal accelerative forces, they will all continue to move among themselves, after the same manner as if they had not been urged by those forces" [1].

Newton described his version of relativity in Corollary V of the Axioms section. He wrote, "The motions of bodies included in a given space are the same among themselves, whether that space is at rest, or moves uniformly forwards in a right line without any circular motion" [1]. This concept of Newton is generalized succinctly [2]:

Newtonian Principle of Relativity: The principles of mechanics that are valid in one coordinate system are equally valid in a coordinate system in motion at constant velocity relative to it; hence on the basis of mechanical phenomena, there is no way of determining absolute velocity; only relative velocity can be measured.

Others have erroneously generalized this concept to include all physical laws. For example, Serway and Jewett stated the first postulate of special relativity is "1) The laws of physics must be the same in all inertial reference frames." [3, p. 1223]. Also, Young, Adams and Chastain wrote: "All laws of physics, including mechanics and electromagnetism, are the same in all inertial frames of reference. In other words, all inertial reference frames are equivalent. Any one is as good as another." [4, p. 861] Unfortunately, light follows the laws of electromagnetism, which is expressed by Maxwell's equations. Electromagnetism does not obey Newton's second law, which force is the derivative of momentum. For example, one component of the electromagnetic Lorentz force is velocity dependent in the cross product term $q \mathbf{V} \times \mathbf{B}$, where $\mathrm{q}$ is the charge, $\mathbf{V}$ is the velocity vector of that charge and $\mathbf{B}$ is the magnetic induction vector of the surrounding field. The velocity in inertial frame 1 must be added to the relative velocity of the second inertial frame to get the correct value of the total velocity of the charged particles mapped in the second inertial frame. However, the fact that electromagnetism does not obey Newton's second law is the property used to determine the relative speed between moving inertial frames. The roundtrip speed of light in moving inertial frames is the same one-way speed of light in an absolutely stationary, nongravitated reference frame [5]. All one has to do is measure the one-way speed of light in a moving inertial frame to determine that inertial frame's velocity relative to an absolutely stationary frame.

The author emphasizes that Newton's original concept involved bodies, but the generalized Newtonian Principle of Relativity involves all mechanical phenomena including heat, pressure and sound. For example, the pressure exerted by an ideal gas has been accurately confirmed by observations of the Brownian motion of fine particles executing random motions due to collisions with fluids by the Maxwellian distribution of individual molecule speeds within the fluid [2, p. 357-358]. One mechanical property of waves is energy can be transferred from one point to another without the physical transfer of material between the points. There is a momentary displacement of a molecule within the fluid that collides with a neighboring molecule to create an overall disturbance that travels through the fluid and transmits energy, but the average displacement of each molecule is zero in Brownian motion, such that there is no physical transfer of the molecule within the fluid. Such disturbances include sound, which is a mechanical wave motion within an elastic medium that can be an ideal solid, liquid or gas. A sound wave requires a source to initiate the mechanical disturbance and an elastic medium to transmit the disturbance. Any noise does not involve the displacement of the elastic medium itself. Sound is the continual succession of disturbances or pulses propagated through the medium. The simplest and important type of successive sound waves is compression wave motion. The distance between such successive waves is the wavelength, $\lambda$, and, if the successive wavelengths are constant through a uniform elastic medium, then the sound has a steady frequency, f, relative to the speed of sound, $v$, through the uniform medium by the formula $v=f \lambda$.

Newton's first law requires that velocities must satisfy vector addition. Suppose an observer at rest has two bodies A and B moving uniformly, but in oblique directions. The observer describes the two bodies by velocity vectors $\vec{V}_{\mathrm{AO}}$ and $\vec{V}_{\mathrm{BO}}$. The opposite velocity vector, $\overrightarrow{\mathrm{V}}_{\mathrm{OA}}=-\overrightarrow{\mathrm{V}}_{\mathrm{AO}}$, describes the motion of the observer relative to body $\mathrm{A}$. The velocity of $B$ relative to $A$ is the vector addition as required by Newton's Corollary I by replacing force vectors with velocity vectors so that $\vec{V}_{\mathrm{BA}}=\vec{V}_{\mathrm{BO}}-\vec{V}_{\mathrm{AO}}=\vec{V}_{\mathrm{BO}}+\overrightarrow{\mathrm{V}}_{\mathrm{OA}}$. Vector addition of velocities is required to translate the velocities of moving uniform bodies relative to an inertial observer into the inertial frame attached to one of the inertial bodies.

The ideal medium, which has no internal average movement of molecules from turbulences or vortices, is effectively an inertial system. For example, the speed of sound through still air is horizontally the same in all directions. (The author is excluding the vertical speed of sound due to changing air density with altitude until there is no atmosphere in outer space to transmit sound and is assuming a uniform temperature and pressure for air.) If an observer is standing on the ground, the speed of sound, $\vec{V}_{\mathrm{s}}$, is the same in still air. If there is a constant horizontal wind, the effective speed of sound varies directionally for the observer according to the velocity addition formula, $\vec{V}_{\text {eff }}=\vec{V}_{s}+\vec{V}_{\text {wind }}$. When the observer uniformly moves relative to the ground through still air, then the velocity of the observer is effectively a constant wind in the opposite direction such that 
$\vec{V}_{\text {obs }}=-\vec{V}_{\text {wind }}$. Only in the ideal medium is the speed of sound a constant in all directions, but the effective sound velocity varies directionally due to the relative velocity between the medium and observer. This was elaborated in a separate paper by the author with a generalized formula for the Doppler effect [6].

The speed of light is a constant in all directions within the domain of an absolutely stationary, nongravitated reference frame. Since the velocity of light in a vacuum is large, but finite, the relative velocity between the absolutely stationary frame and the moving observer can be determined from oneway timed measurements of light traversing a fixed length L. This is similar to determining the relative velocity between the air and observer by direct velocity measurements of sound in different directions. This is covered in the next section.

\section{Measuring Velocities of Moving Inertial Frames Using Light}

As stated earlier, it is assumed that the speed of light is constant in every direction in the domains of absolutely stationary, nongravitated reference frames (i.e. resting frames). This assumption is not as restrictive as the general claim that the speed of light is a universal constant in a vacuum in all directions in all inertial frames. Even though it is based on the similarity of sound speeding through an ideal, uniform medium, it is immaterial whether ether exists to transmit light, only that light has a constant speed in a particular direction of a resting frame. The objective is to use light in one-way measurements to determine the velocity of the moving inertial frame relative to a resting frame. A laboratory on Earth is assumed sufficiently inertial if the three-dimensional measurements are taken simultaneously.

The test setup will time the one-way light transmission over a fixed distance L. A uniformly moving rigid rod of length $\mathrm{L}$ has endpoints $\mathrm{A}$ and $\mathrm{B}$. From the perspective of the resting frame, light is transmitted by a moving rod's end in the resting frame (e.g. the origin) at coordinate time $t$, and the rod moves collinearly with a constant velocity $\mathrm{v}$. The series was derived for computing the distance $\mathrm{D}$ when the light reached the opposite endpoint of the moving rod [5,7]. Let $\mathrm{AB}$ designate the emitted light from $\mathrm{A}$ overtaking the receding endpoint $\mathrm{B}$ and let $\mathrm{BA}$ designate the emitted light from B meeting the oncoming endpoint A that light would travel:

$$
\mathrm{L}_{\mathrm{AB}}=\frac{\mathrm{L}}{1-\mathrm{v} / \mathrm{c}}=\mathrm{L}\left(1+\frac{\mathrm{v} / \mathrm{c}}{1-\frac{\mathrm{v}}{\mathrm{c}}}\right) \text { for the receding endpoint, and }
$$

$$
\mathrm{L}_{\mathrm{BA}}=\mathrm{L}\left(1-\frac{\mathrm{v} / \mathrm{c}}{1-\frac{\mathrm{v}}{\mathrm{c}}}\right) \text { for the approaching endpoint. }
$$

To calculate the transmission interval for either case, divide by $\mathrm{c}$ where $\mathrm{T}=\mathrm{L} / \mathrm{c}$ in an absolutely stationary frame.

$$
\begin{gathered}
\Delta \mathrm{T}_{\mathrm{AB}}=\frac{\mathrm{L}}{\mathrm{c}}\left(1+\frac{\mathrm{v} / \mathrm{c}}{1-\frac{\mathrm{v}}{\mathrm{c}}}\right)=\mathrm{T}\left(1+\frac{\mathrm{v} / \mathrm{c}}{1-\frac{\mathrm{v}}{\mathrm{c}}}\right), \text { and } \\
\Delta \mathrm{T}_{\mathrm{BA}}=\frac{\mathrm{L}}{\mathrm{c}}\left(\frac{1-2 \mathrm{v} / \mathrm{c}}{1-\mathrm{v} / \mathrm{c}}\right)=\mathrm{T}\left(1-\frac{\mathrm{v} / \mathrm{c}}{1-\frac{\mathrm{v}}{\mathrm{c}}}\right) .
\end{gathered}
$$

The effective parallel and antiparallel velocities of light relative to the uniformly moving length $\mathrm{L}$ are obtained by dividing the length $\mathrm{L}$ by the time it takes light to traverse that length.

$$
\mathrm{C}_{\mathrm{AB}}=\frac{\mathrm{L}}{\frac{\mathrm{L}}{\mathrm{c}\left(1+\frac{\mathrm{v}}{\mathrm{c}}\right)}=\mathrm{c}\left(\frac{1-\frac{\mathrm{v}}{\mathrm{c}}}{1}\right)}=\mathrm{c}\left(\frac{\mathrm{c}-\mathrm{v}}{\mathrm{c}}\right)=\mathrm{c}-\mathrm{v}
$$

$$
\mathrm{C}_{\mathrm{BA}}=\frac{\mathrm{L}}{\frac{\mathrm{L}}{\mathrm{c}}\left(1-\frac{\frac{\mathrm{v}}{\mathrm{c}}}{1-\frac{\mathrm{v}}{\mathrm{c}}}\right)}=\mathrm{c}\left(\frac{1-\frac{\mathrm{v}}{\mathrm{c}}}{1-\frac{2 \mathrm{v}}{\mathrm{c}}}\right)=\mathrm{c}\left(1+\frac{\frac{\mathrm{v}}{\mathrm{c}}}{1-\frac{2 \mathrm{v}}{\mathrm{c}}}\right) \approx \mathrm{c}\left(1+\frac{\frac{\mathrm{v}}{\mathrm{c}}}{1}\right)=\mathrm{c}+\mathrm{v} \text { if } \mathrm{v}<<\mathrm{c}
$$

This shows that the effective one-way velocity of light in the moving inertial frame obeys vector addition of velocities. Once the time interval $\Delta \mathrm{T}$ is measured, the parallel velocity to the length $\mathrm{L}$ is calculated by either of these two formulas:

$$
\begin{aligned}
& \mathrm{v}=\mathrm{c}\left(1-\frac{\mathrm{T}}{\Delta \mathrm{T}}\right) \text { when } \Delta \mathrm{T} \geq \mathrm{T}=\mathrm{L} / \mathrm{c} \\
& \mathrm{v}=\mathrm{c}\left(\frac{\mathrm{T}-\Delta \mathrm{T}}{2 \mathrm{~T}-\Delta \mathrm{T}}\right) \text { when } \Delta \mathrm{T} \leq \mathrm{T}=\mathrm{L} / \mathrm{c}
\end{aligned}
$$

Any laboratory measuring the time interval $\Delta \mathrm{T}$ is noninertial, as Earth's four ocean tides demonstrate that Earth is accelerated. However, the velocity of the moving Earth compared to a resting frame is expected to be small and the transmission time $\Delta \mathrm{T}$ should be in nanoseconds when $\mathrm{L}$ is a few meters long. Thus, to a good approximation, the laboratory frame should be sufficiently inertial in mathematical derivations. The measured round-trip light speed in the moving inertial frame is equivalent to the one- way light speed (e.g. $\mathrm{c}=299792458 \mathrm{~m} / \mathrm{s}$ ) in the resting (i.e. nongravitated, absolutely stationary) frame [5, 7]. Equations (7) and (8) determine the one-dimensional velocity in the $X$, $\mathrm{Y}$ or $\mathrm{Z}$ axis of the laboratory frame, and $\Delta \mathrm{T}$ can be compared directly to the expected $\mathrm{T}=\mathrm{L} / \mathrm{c}$ when $\mathrm{L}$ is precisely measured.

The three-dimensional absolute velocity of the moving laboratory frame will have to be measured in all reference axes simulatneously. It is recommended to set the perpendicular $\mathrm{X}$ - and $\mathrm{Y}$-axis in the locally horizontal plane and the $\mathrm{Z}$-axis gravitationally vertical and oriented by the right hand rule. If the length $\mathrm{L}$ is very precisely measured (e.g. using adjustable interferometers and counting wavelengths), the vertical velocity may need to be adjusted for gravity. As demonstrated by the Pound-Rebka experiment [8], light's velocity was increased downward or decreased upward by gravity $\mathrm{g}$. As $\delta \mathrm{V}_{\mathrm{z}}=\mathrm{g} \Delta \mathrm{T}_{\mathrm{z}}$, the vertical velocity of the laboratory frame relative to a resting frame is $\mathrm{V}_{\mathrm{z}}=$ 
$\mathrm{V}_{\text {measured }} \pm \delta \mathrm{V}=\mathrm{L}_{\mathrm{z}} / \Delta \mathrm{T} \pm \mathrm{g} \Delta \mathrm{T}$ where \pm applies respectively to an upward or downward transmission.

A minimum of a year is needed to collect timed measurements to determine the laboratory's threedimensional velocity relative to a resting frame, such as the celestial sphere. Dynamical astronomy is needed to rotate the laboratory frame to the celestial sphere due to polar wobble, Earth rotation, nutation, and precession, besides the total velocity changes from Earth's orbital velocity around the Sun of approximately $29.8 \mathrm{~km} / \mathrm{s}$, the Sun's orbital speed within the rotating Milky Way of $220 \mathrm{~km} / \mathrm{s}$, and the $300 \mathrm{~km} / \mathrm{s}$ velocity of the Milky Way approaching Andromeda [9] [10]. It takes about $3.3 \mathrm{~ns}$ for light to travel 1 stationary meter in vacuum as $\mathrm{c} \approx 3 \mathrm{E} 8 \mathrm{~m} / \mathrm{s}$. So, the extra time for the one-way velocity of light to travel a meter moving parallel with Earth's orbital velocity is $\pm 3.3 \mathrm{E}-13$ s by equations (3) or (4), which would require a precision of about 1 fs for results obtaining three significant figures.

Many different test configurations are possible that are equally valid to measure the one-way speed of light, so test equipment and setup are only outlined. The basic suggested design consists of a precise time mechanism to trigger three low powered lasers to send light pulses at photocells located at calibrated distances parallel to the laboratory's coordinate axes and time the return signal through separate circuitries with the same timing device. To get consistent timed measurements at fs precision, an ultraprecise hydrogen maser of $1 \mathrm{E}-15 \mathrm{~s} / \mathrm{s}$ uncertainty is recommended for its short and long term stability of operation. Although the definition of the atomic second involves cesium clocks, those devices have short-term random excursions that take about a day to average out. One electronic pulse, which is time tagged by the timing device, will trigger a pulse from each laser through individual circuits to its facing photocell, which sends an electronic signal back to the timing mechanism to record the round trip transmission. The electric signals are electrons conveyed by the atoms that make up the wires and circuitry fixed relative to the laboratory frame. As Newton's Corollary VI claims [1, p. 19], the Newtonian forces on the laboratory will accelerate the electrons equally and in parallel over the whole circuitry, so the electrons will complete a circuit in the same time as if the laboratory was absolutely stationary. With the laser placed against the photocell, each circuit is calibrated for the round-trip time, $T_{R}$, between the triggering electric pulse and the returning electric signal. Some criteria will need to define the leading edge of each electric signal for timing purposes. Then, move each photocell at a calibrated distance, L, away from the laser to increase the round-trip transmission, which will include the time interval, $\Delta \mathrm{T}$, that light was transmitted over the distance L. As previously discussed, the local vertical acceleration due to Earth's gravity is compensated in the vertical time measurement. It is recommended to have unequal lengths $\mathrm{L}$, so that it is obvious the returning signal is from the appropriate photocell. For example, if $\mathrm{L}_{X}$ is 1 meter, then let $\mathrm{L}_{Y}=\pi / 2$ meters and $\mathrm{L}_{\mathrm{Z}}=$ e meters, so that the returning signals will be about 3, 4.7 and $8.2 \mathrm{~ns}$ apart in irrational ratios to distinguish the leading edges from internal reflections or delays from earlier signals. It is recommended to keep the laboratory under constant temperature and well isolated from vibrations during the data collection over a year and routinely recheck the calibration of each circuit's roundtrip time, $T_{R}$. If the laser transmission is through air, fiber optics, or other media, use the appropriate index of refraction to convert the equivalent light speed in vacuum.

The time intervals, $\Delta \mathrm{T}_{\mathrm{i}}$, in each coordinate will be calculated for equations (7) or (8) for each circuit's recorded round-trip time, $\mathrm{T}_{\mathrm{i}}$, along the $\mathrm{X}$ or $\mathrm{Y}$ axis:

$$
\Delta \mathrm{T}_{\mathrm{i}}=\mathrm{T}_{\mathrm{i}}-\mathrm{T}_{\mathrm{Ri}} \text { where } \mathrm{i}=\mathrm{X} \text { or } \mathrm{Y} .
$$

For the $\mathrm{Z}$ axis, the vertical velocity may need adjusting for Earth's gravity by the following formulae:

$$
\begin{gathered}
\mathrm{v}_{\mathrm{Z}}=\mathrm{c}\left(1-\frac{\mathrm{T}}{\Delta \mathrm{T}_{\mathrm{Z}}}\right) \pm \mathrm{g} \Delta \mathrm{T}_{\mathrm{Z}} \text { when } \Delta \mathrm{T}_{\mathrm{Z}} \geq \mathrm{T}=\mathrm{L} / \mathrm{c} \text { for upward/downward transmission, or } \\
\mathrm{v}_{\mathrm{Z}}=\mathrm{c}\left(\frac{\mathrm{T}-\Delta \mathrm{T}}{2 \mathrm{~T}-\Delta \mathrm{T}_{\mathrm{Z}}}\right) \pm \mathrm{g} \Delta \mathrm{T}_{\mathrm{Z}} \text { when } \Delta \mathrm{T}_{\mathrm{Z}} \leq \mathrm{T}=\mathrm{L} / \mathrm{c} \text { for upward/downward transmission. }
\end{gathered}
$$

The rotation matrices for pole wander, Earth rotation, nutation, and precession will need to be calculated to transform the $3 \mathrm{D}$ velocities from the laboratory frame to some inertial reference frame (such as the celestial sphere or J2000.0).

The evidence for one-way variability in light can be found in the Pound-Rebka experiment [8], which measured the frequency changes. It also confirmed the formula $\delta \mathrm{v}=\mathrm{g}$ $\mathrm{H} / \mathrm{c}$. was correct over short vertical distances, which seems to indicate that light's velocity increased or decreased through local gravity by $\delta \mathrm{v}$ when photons decreased or increased altitude $\mathrm{H}$. Thus, it is expected to measure a 3dimensional velocity variation with one-way speed of light measurements.

\section{Conclusion}

Newton set the foundation of physics with his Mathematical Principles of Natural Philosophy. Besides his three axioms, he held that two simultaneous forces would be the same as the vector sum of the individual forces (Corollary I). Newton's relativity is stated in Corollary VI as "The motions of bodies included in a given space are the same among themselves, whether that space is at rest, or moves uniformly forwards in a right line without any circular motion". The generalized Newtonian Principle of Relativity posits that any general equation of motion or force equation retains the same form for all inertial reference frames, which is true when forces that obey Newton's second law interact 
with bodies. However, equations incorporating velocity must include the mutual velocity between inertial frames for the correct transformation. For example, a stationary mass in one inertial frame has no momentum in that frame, but a second moving inertial frame must assign a nonzero momentum to that mass due to its apparent velocity in the second inertial frame. When no forces interact with a body, the body still can have a constant linear velocity by Newton's first law as a constant of integration. That law requires vector addition of velocities between inertial frames. The vector velocity between inertial frames transforms correctly the equations of motion between inertial frames. Rather than assume the restrictive assumption that the speed of light is universal for all inertial frames, it is assumed the speed of light is a constant in all directions for nongravitated, absolutely stationary frames. As shown in previous papers [5, 7], the one-way speed of light passing a moving, parallel rod is directionally different due to the finite time it takes light to reach the opposite end of the moving rod, which is receding from or approaching toward the light.

A description is provided in the paper for the experimental apparatus and setup to measure the velocity of light in three dimensions over calibrated lengths. The apparatus of lasers and photocells is electrically connected with a hydrogen maser for timing the events between flashing and reception. The electrical design will time the interval between triggering the laser and the electrical return of the photocell's response back to the trigger point. The first step will calibrate the precise transmission relay, $t_{r}$, through each circuit between the triggering pulse and the electrical return (when the laser and photocell are adjacent to each other in a laboratory controlled environment). By Newton's Corollary VI, any external forces that affect all bodies in the same parallel direction will have no effect on the internal interaction between the bodies. So, the time to complete an electrical signal through a circuit is expected to be the same whether that circuit experienced external parallel forces due to the motion of the laboratory frame or whether no Newtonian forces were present. The electrons in the circuitry would be dragged along within the laboratory frame, so the closed circuit transmission delay would be fixed as $\tau_{\text {delay. }}$ Separate the laser and photocell to a rigidly fixed, calibrated distance L. The laser light would be moving independent of the laser source and circuitry. The maser would measure the observed total time interval in that direction as $\tau_{\text {observed. }}$ The author recommends the time measurement be made locally horizontal in the laboratory's x-axis and y-axis, and measure vertically in the $\mathrm{z}$-axis. The respective velocities in each coordinate will be calculated simply as $\tau_{\text {observed }}-\tau_{\text {delay }}=\tau$.
The vertical velocity should be adjusted for the local gravity. The final 3-dimensional velocity for the laboratory frame relative to a resting frame will be the vector sum of the three individual velocities.

If the author's theory is consistent and experimentally verified, it may take years to plot the laboratory's velocity as a moving reference frame relative to an "ideal" resting frame, such as the celestial sphere. This will entail dynamical astronomy to separate Earth's rotation, precession, nutation, polar wobble and orbital velocity (about $29.8 \mathrm{~km} / \mathrm{s}$ [9]) with the solar system's velocity [about $220 \mathrm{~km} / \mathrm{s}[9,10])$ caused by the Milky Way's rotation and by the Milky Way's translational velocity (about $300 \mathrm{~km} / \mathrm{s}[9,10]$ ) in the universe. If this is verified, then coordinate clocks can be properly synchronized to a master clock by incorporating the velocity of the moving inertial frame when the master time is broadcasted to the remote clocks that are located a fixed distance $\mathrm{L}$ from the master clock.

\section{References}

[1] Motte, A., and Cajori, F., 1934 translation of Isaac Newton's Mathematical Principles of Natural Philosophy, Encyclopædia Britannica (copyright 1952), University of California Press, twenty-first printing, 1977.

[2] Shortley, G., and Williams, D., Elements of Physics, 4th edition, Prentice-Hall, Inc. (1965).

[3] Serway, R. A. and Jewett Jr., J. W., Physics for Scientists and Engineers, $9^{\text {th }}$ ed., Cengage Learning (2014).

[4] Young, H. D., Adams, P. W. and Chastain, R. J., Sears and Zemansky's College Physics, Pearson (2016), $10^{\text {th }}$ ed.

[5] Deines, S. D., "Vector Addition of Light Speed Versus the Hafele-Keating Time Dilation Test", to be published in IJAMTP (2017).

[6] Deines, S. D., "Generalized Equations for the Collinear Doppler Effect”, to be published in IJAMTP (2017).

[7] Deines, S. D., "Dichotomy between Length Contraction and Null Results from All Interferometer Experiments", to be published in IJAMTP (2017).

[8] Pound, R. V.; Rebka Jr. G. A., "Gravitational Red-Shift in Nuclear Resonance". Physical Review Letters (November 1, 1959) 3 (9): 439-441.

[9] Fix, J. D., Astronomy: Journey to the Cosmic Frontier, $5^{\text {th }}$ ed., McGraw-Hill Higher Education (2008).

[10] Fix, J. D., Astronomy, $3^{\text {rd }}$ ed., McGraw-Hill Education (2004). 